\title{
AN ORDER RELATION AMONG TOPOLOGICAL SPACES
}

\author{
BY \\ C. N. MAXWELL
}

In this paper we define a partial ordering on a class of topological spaces as follows $\left({ }^{1}\right)$ : For two spaces $X$ and $Y$ in the class, we will say $X \leqq Y$ if for each open cover $\alpha$ of $X$, there is a continuous function $f: X \rightarrow Y$ which is an onto $\alpha$-map. (Recall that $f$ is an $\alpha$-map if there is an open cover $\beta$ of $Y$ such that $f^{-1}(\beta)$ refines $\alpha$.) It is easy to check that the relation $\leqq$ is reflexive and transitive. In [7], Kuratowski proved that the closed $n$-dimensional ball is not $\leqq$ the $n$-sphere. More recently, Ulam [8] raised the question whether the 2-dimensional ball is $\leqq$ the 2-dimensional torus. Ulam's question has stimulated interest in the ordering itself, and a result of T. Ganea [5] states, roughly speaking, that if $X \leqq Y$ and $Y$ is a compact $n$-dimensional manifold and $X$ is an absolute neighborhood retract, then $X$ has the same homotopy type as an $n$-dimensional manifold. This result then gives Ulam's question a negative answer $\left({ }^{2}\right)$, and suggests studying properties of a space $Y$ which are inherited by a space $X$ satisfying $X \leqq Y$.

The purpose of this paper is to present certain properties which are inherited, in the above sense. Also, comparison is made between this ordering and the ordering given by dimension (Theorem 1), and (for the class of absolute neighborhood retracts) the ordering given by $\alpha$-domination for every cover $\alpha$ (Theorem 2). Since the property of possessing a fixed point under every continuous function will be shown to be inherited, another question of Ulam's is answered here [8, Chapter IV, Problem 12].

TheOREM 1. If $X \leqq Y$, then:

(1) If $X$ or $Y$ is compact, then both are compact.

(2) If $X$ or $Y$ is connected, then both are connected.

(3) If $Y$ is paracompact, then $X$ is paracompact.

(4) If $Y$ is Lindelöf, then $X$ is Lindelöf.

(5) Dimension $X \leqq$ dimension $Y$.

(6) If $X$ is $T_{1}$ and $Y$ is Hausdorf, then $X$ is Hausdorff.

(7) If $X$ is $T_{1}$ and $Y$ is regular, then $X$ is regular.

(8) If $X$ is $T_{1}$ and $Y$ is completely regular, then $X$ is completely regular.

(9) If $Y$ is normal, then $X$ is normal.

This paper has been submitted to and accepted for publication by the Proceedings of the American Mathematical Society. It has been transferred to these Transactions, with the consent of the author, for technical reasons. Received by the editors September 27, 1959 and, in revised form, October $1,1960$.

(1) This work was accomplished with the aid of a grant from the University of Alabama Research Committee (Project 372).

(2) The negative answer has also been demonstrated by M. K. Fort, Jr. [4]. 
REMARK. In (5) we are taking, as definition, the dimension of a space $A$ is $\leqq n$ if every open cover of $A$ can be refined by a cover of order $\leqq n$. In case $A$ is paracompact and Hausdorff, then this is equivalent to the condition: Every finite open cover can be refined by an open cover of order $\leqq n$. See $[1 ; 2]$. The proofs of (1), (2), (3), (4), and (5) are almost immediate from the definitions and are omitted. The proofs of (6), (7), (8), and (9) are similar to one another and we prove only (9).

Proof of (9). Suppose $X \leqq Y$ and $Y$ is normal. Let $A$ be closed in $X, W$ open in $X$, and $A \subset W$. Let $\alpha=\{X-A, W\}$. Then there exists $f: X \rightarrow Y$ an onto $\alpha$-map. Now if $x \in f^{-1}\left([f(A)]^{-}\right)$, then there is some open neighborhood $B(x)$ of $f(x)$ such that $f^{-1}(B(x))$ lies in an element of $\alpha$. Hence, either $f^{-1}(B(x))$ $C W$ or $f^{-1}(B(x)) \cap A=\varnothing$. But since $f(x)$ is in $[f(A)]-$ and $B(x)$ is a neighborhood of $f(x)$, we have $B(x) \cap f(A) \neq \varnothing$. Therefore $f^{-1}(B(x)) \cap A \neq \varnothing$ and the second alternative is impossible. So for each $x \in f^{-1}\left([f(A)]^{-}\right)$, the set $B(x)$ is an open neighborhood of $f(x)$ such that $f^{-1}(B(x)) \subset W$. Let

$$
V=U\left\{B(x): x \in f^{-1}\left([f(A)]^{-}\right)\right\} \text {. }
$$

Then $V$ is open in $Y,[f(A)]^{-} \subset V$, and $f^{-1}(V) \subset W$. By normality of $Y$, there exists an open set $U$ such that $[f(A)]-\subset U \subset \bar{U} \subset V$. Then $A \subset f^{-1}(f(A))$ $\subset f^{-1}\left([f(A)]^{-}\right) \subset f^{-1}(U) \subset\left[f^{-1}(U)\right]^{-} \subset f^{-1}(\bar{U}) \subset f^{-1}(V) \subset W$. Let $T=f^{-1}(U)$. Then $A \subset T \subset \bar{T} \subset W$, and $X$ is normal.

Theorem 2. Let $X$ and $Y$ be $\operatorname{ANR}(m e t r i c)$. If $X \leqq Y$, then for each open cover $\alpha$ of $X$, there are maps $f: X \rightarrow Y$ and $g: Y \rightarrow X$ such that:

(1) $f$ is an onto $\alpha-m a p$.

(2) $g f$ is $\alpha$-homotopic to the identity on $X$.

Proof $\left({ }^{3}\right)$. Let $\alpha$ be an open cover of $X$. By Hanner's Theorem [6, Theorem 4.1, p. 395], there is a refinement $\beta$ of $\alpha$ such that if $t: X \rightarrow X$ is any continuous function with the property that for each $x \in X, x$ and $t(x)$ lie in a common element of $\beta$, then $t$ is $\alpha$-homotopic to the identity. Let $\gamma$ be a star refinement of $\beta$. By Dugundji's result [3, Lemma 7.3, p. 364], there exists a refinement $\delta$ of $\gamma$ such that any partial realization of a CW complex $P$ in $X$ relative to $\delta$ can be extended to a full realization of $P$ relative to $\gamma$.

Using the hypothesis $X \leqq Y$, there exists $f: X \rightarrow Y$ onto $Y$ and an open cover $\epsilon$ of $Y$ such that $f^{-1}(\epsilon)$ refines $\delta$. Let $\eta$ be a star refinement of $\epsilon$. By Dugundji's Theorem [3, Theorem 7.5, p. 365], there is a simplicial complex $P$ and mappings $g^{\prime}: Y \rightarrow P$ and $h: P \rightarrow Y$ such that $h g^{\prime}$ is $\eta$-homotopic to the identity on $Y$, and, for each cell $\sigma$ of $P, h(\sigma)$ lies in some element of $\eta$.

Let $Q$ be the set of vertices of $P$. Since $f$ is onto, we can find for each $q \in Q$ an element $x \in X$ such that $f(x)=h(q)$. Define $m: Q \rightarrow X$ by $m(q)=$ any $x \in X$ for which $f(x)=h(q)$. Then $m$ is a partial realization of $P$ in $X$ relative

(3) The author wishes to express his gratitude to the referee for improvements suggested concerning this theorem. 
to $\delta$. For if $q_{0}, \cdots, q_{n}$ determine a cell of $P$, then $h\left(q_{0}\right), \cdots, h\left(q_{n}\right)$ lie in a single element of $\eta$. But $\eta$ refines $\epsilon$ and $f^{-1}(\epsilon)$ refines $\delta$. Hence the sets $f^{-1}\left(h\left(q_{0}\right)\right), \cdots, f^{-1}\left(h\left(q_{n}\right)\right)$ lie in a single element of $\delta$. Thus, $m$ can be extended to a full realization $l$ of $P$ relative to $\gamma$.

Now define $g: Y \rightarrow X$ by $g=l g^{\prime}$. We want to verify that for each $x \in X$, $g f(x)$ and $x$ lie in a common element of $\beta$. Take $x \in X$. Then $f(x)$ and $h g^{\prime} f(x)$ lie in some element $H_{1}$ in $\eta$ since $h g^{\prime}$ is $\eta$-homotopic to the identity. Let $\sigma$ be the cell of $P$ containing $g^{\prime} f(x)$ and $v$ be a vertex of $\sigma$. Let $l(v)=x^{\prime}$, and thus $f\left(x^{\prime}\right)=h(v)$. Since $h(\sigma)$ lies in an element $H_{2}$ of $\eta, h g^{\prime} f(x)$ and $h(v)$ are in $H_{2}$. Therefore $h g^{\prime} f(x) \in H_{1} \cap H_{2}$. But since $\eta$ is a star refinement of $\epsilon$, there is a set $E \in \epsilon$ such that $f(x), h g^{\prime} f(x)$, and $h(v)=f\left(x^{\prime}\right)$ are all in $E$. Therefore, $x$ and $x^{\prime}$ are in $f^{-1}(E)$. But $f^{-1}(\epsilon)$ refines $\delta$. Hence there exists a set $D_{1}$ in $\delta$ such that $x$ and $x^{\prime}=l(v)$ are in $D_{1}$. But $g^{\prime} f(x)$ and $v$ are in $\sigma$ and $l g^{\prime} f(x)$ and $l(v)$ are in $l(\sigma)$. That is, $g f(x)$ and $x^{\prime}$ are in $l(\sigma)$. Since $l$ is a realization of $P$ in $X$ relative to $\gamma$, there exists $C_{2}$ in $\gamma$ such that $g f(x)$ and $x^{\prime}$ are in $C_{2}$. Let $C_{1}$ be a set in $\gamma$ containing $D_{1}$. Then $x^{\prime} \in C_{1} \cap C_{2}$. Hence $C_{1} \cup C_{2} \subset B$ for some $B \in \beta$. Therefore $g f(x)$ and $x$ are in a single set in $\beta$, and hence $g f$ is $\alpha$-homotopic to the identity.

THEOREM 3. If $X$ and $Y$ are ANR(metric) and $X \leqq Y$ and $Y$ has the property that every continuous function from $Y$ into $Y$ has a fixed point, then $X$ has the fixed point property also.

Proof. Suppose $k: X \rightarrow X$ is continuous without fixed points. Then we can find an open cover $\alpha$ of $X$ which is free with respect to $k$, that is $A \cap k^{-1}(A)=\varnothing$ for all $A$ in $\alpha$. Let $\beta$ be a star refinement of $\alpha$; then $\beta$ is also free with respect to $k$. Using the previous theorem, there are maps $f: X \rightarrow Y$ and $g: Y \rightarrow X$ such that $f$ is an onto $\beta$-map and $g f$ is $\beta$-homotopic to the identity on $X$. Let $h: Y \rightarrow Y$ be defined by $h=f k g$ and let $y$ be a fixed point of $h$. Since $f$ is onto, there exists $x \in X$ such that $f(x)=y$. Hence,

$$
h(y)=h(f(x))=f k g f(x)=f(x)=y .
$$

Since $k g f(x)$ and $x$ are mapped into the same point by $f$, there is a set $B_{1} \in \beta$ such that $k g f(x)$ and $x$ are both in $B_{1}$. Since $g f$ is $\beta$-homotopic to the identity on $X$, there exists $B_{2} \in \beta$ such that $g f(x)$ and $x$ are both in $B_{2}$. Therefore $B_{1} \cap B_{2}$ contains $x$, and there exists $A \in \alpha$ such that $k g f(x), x$, and $g f(x)$ are all in $A$. Hence, $g f(x) \in A \cap k^{-1}(A)$, contradicting the assumption that $\alpha$ is free with respect to $k$.

REMARKS. In case $X$ and $Y$ are compact metric spaces, the relation $X \leqq Y$ can be characterized as follows: For each positive real number $\epsilon$, there is a continuous function $f: X \rightarrow Y$ onto $Y$ for which the diameter of $f^{-1}(y)<\epsilon$ for all $y \in Y$. Using this, we construct some examples.

(1) Let $X$ be the closed interval $[0,1]$. Let $A$ and $B$ be the subsets of the plane defined by $A=\left\{(x, y): x^{2}+y^{2}=1\right\}$ and $B=\{(x, y): y=0$ and $1 \leqq x \leqq 2\}$. 
Let $A \cup B=Y$. Then $X \leqq Y$, for given any $\epsilon>0$ we can map $[0, \epsilon]$ around the circle $A$ so that $f(0)=f(\epsilon)$, and then map $[\epsilon, 1]$ linearly onto $B$. This example shows that $X$ and $Y$ need not have the same homotopy type.

(2) Let $X$ and $B$ be as in example (1). Let $C=\left\{(x, y): x^{2}+y^{2} \leqq 1\right\}$ and $Y=B \cup C$. Then $X \leqq Y$, for given $\epsilon>0$, let $f \operatorname{map}[0, \epsilon]$ onto $C$ so that $f(\epsilon)$ $=(1,0)$ and let $f$ map $[\epsilon, 1]$ linearly on $B$. This example shows that $X$ and $Y$ need not have the same dimension.

\section{REFERENCES}

1. J. Dieudonné, Une generalisation des espaces compacts, J. Math. Pures Appl. Ser. 9 vol. 23 (1944) p. 66.

2. C. H. Dowker, Mapping theorems for non-compact spaces, Amer. J. Math. vol. 69 (1947) p. 211.

3. J. Dugundji, An extension of Tietze's theorem, Pacific J. Math. vol. 1 (1951) pp. 353-367.

4. M. K. Fort, Jr., Epsilon maps of a disk onto a torus, Bull. Acad. Polon. Sci. Sér. Sci. Math. Astr. Phys. vol. 7 (1959) pp. 51-54.

5. T. Ganea, Epsilon mappings onto manifolds, Fund. Math. vol. 47 (1959) pp. 35-44.

6. O. Hanner, Some theorems on absolute neighborhood retracts, Ark. Mat. vol. 1 (1951) pp. 389-408.

7. C. Kuratowski, Sur les transformations des sphères en des surfaces spheriques, Fund. Math. vol. 20 (1933) pp. 206-213.

8. S. M. Ulam, $A$ collection of mathematical problems, New York, Interscience Publishers, 1960.

UNiversity of Alabama,

University, Alabama 